\title{
Optimized design and data analysis of tag-based cytosine methylation assays
}

\author{
Masako Suzuki, Qiang Jing, Daniel Lia, Marién Pascual, Andrew McLellan and John M Greally*
}

\begin{abstract}
Using the type III restriction-modification enzyme EcoP15I, we isolated sequences flanking sites digested by the methylation-sensitive Hpall enzyme or its methylation-insensitive Mspl isoschizomer for massively parallel sequencing. A novel data transformation allows us to normalise Hpall by Mspl counts, resulting in more accurate quantification of methylation at $>1.8$ million loci in the human genome. This HELP-tagging assay is not sensitive to sequence polymorphism or base composition and allows exploration of both CG-rich and depleted genomic contexts.
\end{abstract}

\section{Background}

Epigenetic mechanisms of transcriptional regulation are increasingly being studied for their potential influences in human disease pathogenesis. Much of this interest is based on the paradigm of neoplastic transformation, in which epigenetic changes appear to be universal, widespread throughout the genome, causative of critical transcriptional changes and predictive of disease prognosis (reviewed in [1]). Furthermore, these epigenetic changes represent potential pharmacological targets for reversal and amelioration of the disease process [2].

Of the large number of regulatory processes referred to as epigenetic, there exist numerous assays to study chromatin component distribution, cytosine methylation and microRNA expression genome-wide. The chromatin components include a large number of post-translational modifications of histones, variant histones, DNA-binding proteins and associated complexes, all tested by chromatin immunoprecipitation (ChIP) approaches coupled with microarray hybridization or massively parallel sequencing (MPS). MicroRNAs can be identified and quantified by using microarrays and MPS, while cytosine methylation can be definitively studied by converting the DNA of the genome using sodium bisulfite, shotgun sequencing the product using MPS and mapping this back to the genome to count how frequently cytosines remain unconverted, indicating their methylation in the

*Correspondence: john.greally@einstein.yu.edu

Department of Genetics (Computational Genetics), Center for Epigenomics, Albert Einstein College of Medicine, 1301 Morris Park Avenue, Bronx, NY 10461, USA

() 2010 Suzuki et al.; licensee BioMed Central Ltd. This is an open access article distributed under the terms of the Creative Commons Attribution License (http://creativecommons.org/licenses/by/2.0), which permits unrestricted use, distribution, and reproduction in any medium, provided the original work is properly cited starting material, due to the resistance of methylcytosine to bisulfite conversion compared with unmethylated cytosines. This allows nucleotide resolution, strand-specific, quantitative assessment of cytosine methylation, with such studies performed in Arabidopsis [3-5] and human cells to date [6].

While this approach represents the ideal means of testing cytosine methylation, the amount of sequencing necessary (for the human genome, over 1 billion sequences of $\sim 75$ bp each [6]) to generate quantitative information genome-wide remains prohibitive in terms of cost, limiting these studies to the few referred to above. When studying human disease, the emphasis remains on cytosine methylation assays, as it is generally easier to collect clinical samples for DNA purification than for ChIP or even RNA assays. However, the cell populations harvested are rarely of high purity, and we generally do not know the degree of change in cytosine methylation in the disease of interest and thus the quantitative discrimination required for an assay, with some studies to date indicating that the changes may be quite subtle [7]. These concerns emphasize the need for cytosine methylation assays that can detect methylation levels intermediate in value and changes in disease that are relatively modest in magnitude. Certain microarray-based assays to study cytosine methylation have addressed this issue, with the methylated DNA immunoprecipitation (meDIP) assay amenable to such quantification when used for $\mathrm{CpG}$ islands [8] and possibly also for less CG dinucleotide-rich regions [9]. Restriction enzyme-based assays used with microarrays have also proven to be reasonably quantitative, whether based on methylation-sensitive (for exam- 
ple, the HELP assay [10]) or methylation-dependent (for example, MethylMapper [11]) enzymes. A promising new MPS-based assay is reduced representation bisulfite sequencing (RRBS), which is designed to study the CGdense regions defined by short MspI fragments, and provides nucleotide resolution, quantitative data [12].

The use of MPS for what were previously microarraybased assays has been associated with improved performance [13], as we found when we modified our HELP (HpaII tiny fragment Enrichment by Ligation-mediated PCR) assay [10] for MPS, creating an assay similar to Methyl-Seq [14]. The strength of the HELP assay involves the comparison of the HpaII with the methylation-insensitive MspI representation, allowing a normalization step that makes the assay semi-quantitative [10]. The HELP representation approach was improved upon by Ball et al. [15], who developed the Methyl-Sensitive Cut Counting (MSCC) assay, which involves digesting DNA with HpaII, ligating an adapter to the cohesive end formed, using a restriction enzyme site within the adapter to digest at a flanking sequence and thus capturing the sequence immediate adjacent to the HpaII site. By adding a second MPS-compatible adapter, a library can be generated for MPS, allowing the counting of reads at these sites to represent the degree of methylation at the site. The authors demonstrated the assay to be reasonably quantitative, testing over 1.3 million sites in the human genome, representing not only HpaII sites clustered in CG-dense regions of the genome (approximately $12 \%$ of all HpaII sites are located in annotated CpG islands in the human genome [16]) but also the remaining majority of the genome in which CG dinucleotides are depleted, a genomic compartment not tested by RRBS as currently designed. A focus on the CG-dense minority of the genome will fail to observe changes such as those at CGdepleted promoters (such as OCT4 [17]) and CpG island shores [18], and within gene bodies where cytosine methylation has been found to be positively correlated with gene transcription [15]. It is likely, therefore, that an assay system that can study both CG-dense and CG-depleted regions will acquire substantially more information about epigenomic states than those directed at the CG-dense compartment alone.

In the current study, we tested whether the use of an MspI control would improve MSCC assay performance, as we had found for microarray-based HELP, and whether we could develop an analytical pipeline for routine use of this assay in epigenome-wide association studies. We also explored the use of longer tags than those employed in the MSCC, and added T7 RNA polymerase and reverse transcription steps to allow the generation of libraries without contaminating products, thus obviating the need for gel extraction. The influence of base composition and fragment length parameters as potential sources of bias were also tested, using the H1 (WA01) human embryonic stem (ES) cell line. The outcome is a modified assay that combines the strengths of MSCC and HELP-seq/Methylseq, and the supporting analytical workflow that maximizes the quantitative capabilities of the data generated.

\section{Results and discussion}

\section{Library preparation and sequencing}

We generated HELP tagging libraries with HpaII- or MspI-digested DNA derived from human ES cells using the experimental approach shown in Figure 1. The assay differs from MSCC [15] by using EcoP15I instead of MmeI, generating longer flanking sequences (27 as opposed to 18 to $19 \mathrm{bp}$ ) and the addition of a T7 polymerase and reverse transcription step to allow the generation of the library without contaminating single-adapter products, while in addition obviating the need for gel extraction. After the library preparation, a single band of $125 \mathrm{bp}$ in length was generated, as expected. Libraries

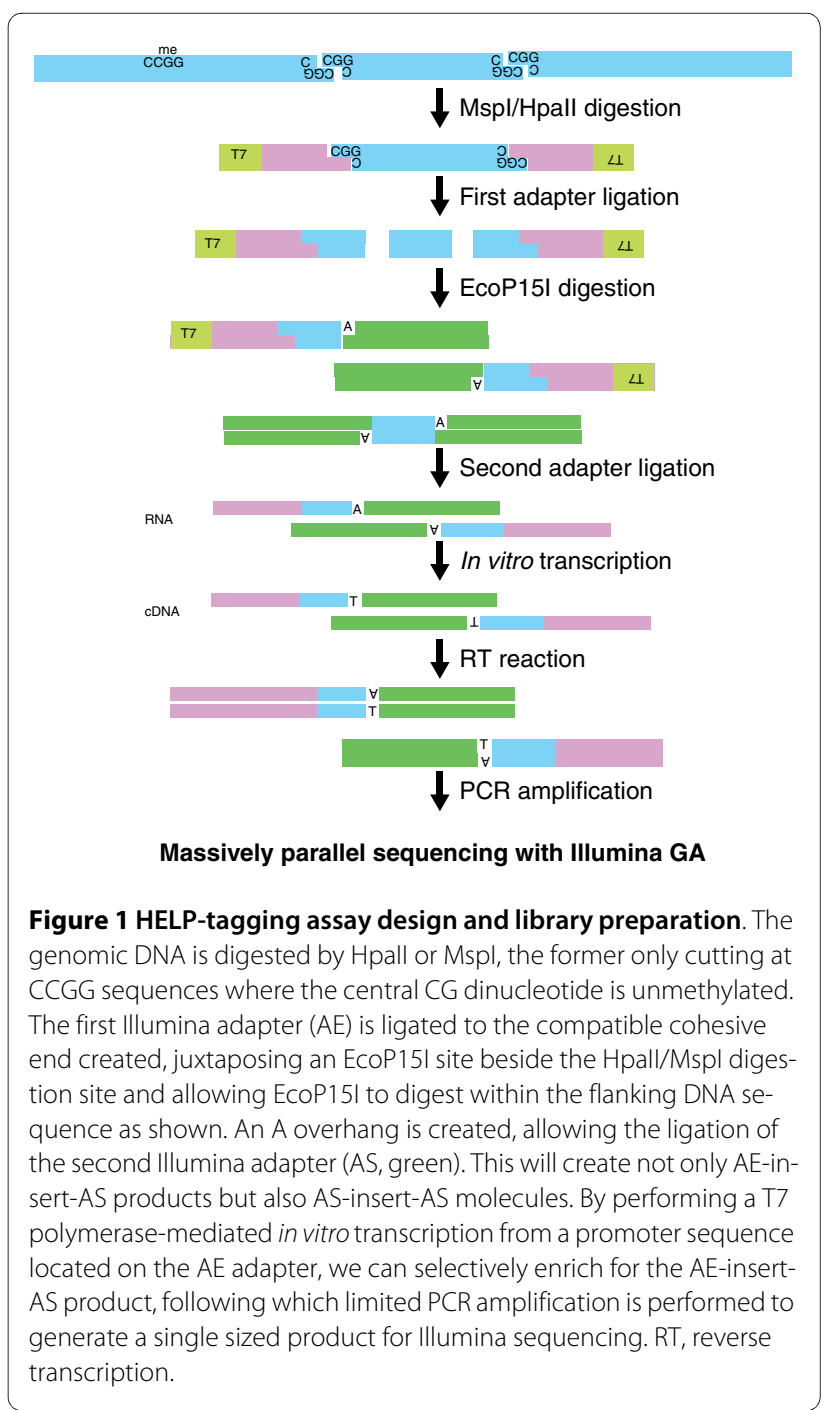


(a)

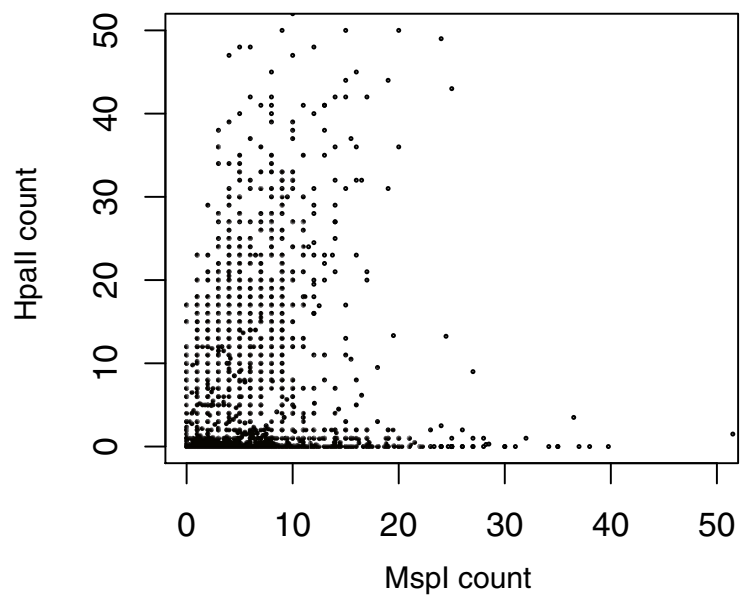

(b)

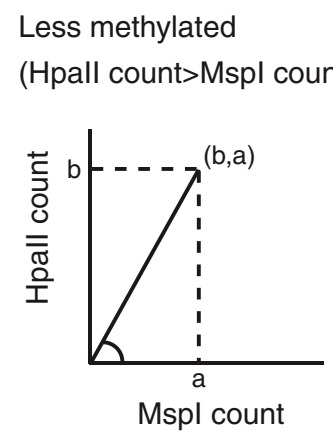

(c)

\section{More methylated}

(Hpall count $<$ Mspl count)

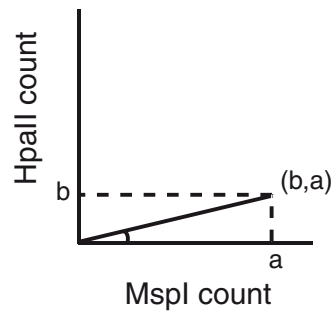

Angle $B=$ degree $(\arctan 2(b, a))$ Length $c=\sqrt{a^{2}+b^{2}}$

(d)

Hpall counts

\section{Angle}
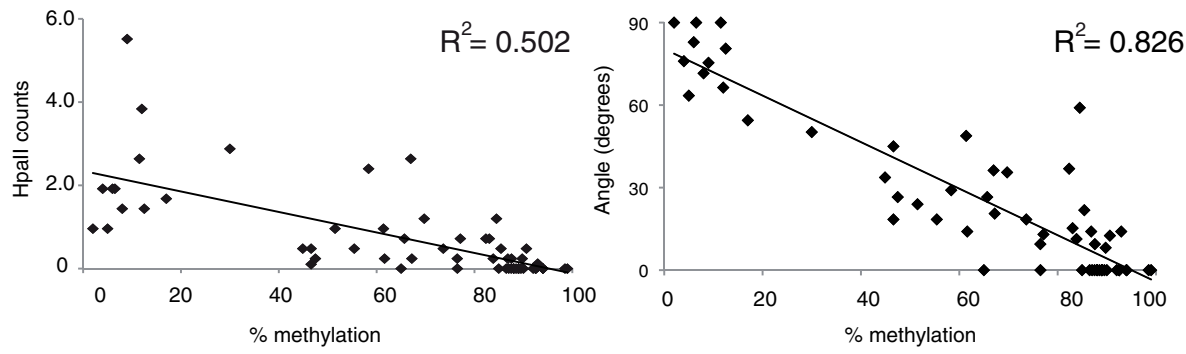

Figure 2 Data transformation and bisulfite validation. (a) Scatter plot showing the relationship between the number of Hpall and Mspl reads at each locus. (b) The location of the data point on the scatter plot indicates whether it is likely to be less or more methylated with larger or smaller angles B subtended as shown, while the confidence of the measurement will be greater when more reads represent the data point, represented by the length of line c. (c) The Hpall count correlates negatively with the degree of methylation, with more counts occurring at loci with less methylation. (d) Transformation of the data to the B angle measure to normalize Hpall by Mspl counts substantially improves the correlation with bisulfite MassArray validation data. 
were sequenced using an Illumina Genome Analyzer (36 bp single end reads) and the sequences were analyzed and aligned using Illumina pipeline software version 1.3 or 1.4. A summary of the Illumina analysis results for each replicate is shown in Table S1 in Additional file 1.

\section{Data quality and reproducibility}

Based on our experimental design, successfully generated products would be expected to possess a 5'-CGCTGCTG sequence at the 3 ' end of the read, the first two nucleotides (CG) representing the cohesive end for ligation of HpaII/MspI digestion products, the remaining six nucleotides the EcoP15I restriction enzyme recognition site. In order to evaluate the yield of desired products, we counted the number of reads containing this sequence and plotted the starting positions of this sequence within the reads obtained. We observed that approximately twothirds of the reads contained the expected sequence, and found that the majority was located at base positions 25 and 26, consistent with the known digestion properties of the restriction enzyme [19]. Removal of the approximately $30 \%$ of reads lacking the CG-EcoP15I sequence was performed to eliminate spurious sequences. In order to investigate sequence quality further, we also determined the number and relative position of Ns (ambiguous base calls) within the reads obtained. Overall, few reads were found to contain Ns, and where they were present, they were found to be evenly distributed by position within the sequence. To test data reproducibility, we compared the results of three experimental replicates against each other using the Pearson correlation coefficient metric. The results of this study showed that all replicates were highly correlated (all the $r$ values exceed 0.9), which confirmed that the technical reproducibility of this assay was excellent (Table S2 in Additional file 1).

\section{Distribution of $\mathrm{Mspl} / \mathrm{Hpall}$ sequence tags}

We merged three lanes of MspI data and observed that approximately $80 \%$ of the $2,292,198$ annotated HpaII sites in the human genome (hg18) were represented by at least one read, for a total of over 1.8 million loci throughout the genome. The mean numbers of reads per locus for MspI and HpaII were 3.94 and 1.82, respectively, and MspI counts were distributed evenly across all genomic compartments examined (Table S3 in Additional file 1). We hypothesize that a combination of incomplete genomic coverage and polymorphisms within some CCGG sites (as we have previously observed [10]) accounts for the $20 \%$ of HpaII sites that were not represented by any reads.

\section{Normalization of Hpall by Mspl counts and data transformation}

When we plot the MspI count on the $\mathrm{x}$-axis and HpaII count on the y-axis for each HpaII site, we can see two major groups of values in the plot (Figure 2a), separated into loci with high or with minimal HpaII counts. This plot helped us to develop a new method for normalizing HpaII counts in terms of variability of the MspI representation. We recognize that hypomethylated loci are associated with relatively greater HpaII counts and a larger angle B (Figure 2b, left) whereas methylated loci will be defined by smaller angle values (Figure $2 b$, middle). Furthermore, some loci will tend to be sequenced more readily than others, and may have identical $B$ values but differing distances from the origin (c distance), allowing a confidence score for identical methylation values (B) in terms of the $c$ distance values (Figure $2 b$, right). To test this model, we used bisulfite MassArray to test quantitatively the cytosine methylation values for $61 \mathrm{HpaII}$ sites (Tables S4, S5 and S6 in Additional file 1), choosing loci representing all components of the $\mathrm{B}$ angle spectrum of values. In Figure 2c, $d$ we show the correlations between these gold standard cytosine methylation values and raw HpaII counts or B angle values. We find that there is the same negative correlation $\left(R^{2}=0.502\right)$ between HpaII counts and cytosine methylation values as demonstrated in the MSCC technique [15], and that the angular transformation of the data incorporating the MspI normalization substantially improves this correlation $\left(R^{2}=0.826\right)$, defining the optimal approach for processing of these data. We represent the data for University of California Santa Cruz (UCSC) genome browser visualization as wiggle tracks, with higher $B$ angle values defining less methylated loci. Methylated loci with zero values that would be otherwise difficult to visualize as having been tested are represented as small negative values. We show the details of the analytical workflow in Figure 3 and an example of a UCSC genome browser representation of HELP-tagging data in Figure S1 in Additional file 1. All data are available through the Gene Expression Omnibus database (accession number [GEO:GSE19937]) and as UCSC genome browser tracks [20].

\section{Potential sources of bias: base composition and fragment length}

As the number of reads at CCGG sites following MspI digestion should not be influenced by methylation, the representation obtained from MspI digestion allowed us to look for systematic sources of bias inherent to the assay. A major concern was that base composition could be a source of such bias, as it has been reported that Illumina sequencing can be influenced by GC composition [21], possibly because of the gel extraction step [22]. Our protocol does not require gel extraction and only begins to show an under-representation of sequences when the $(\mathrm{G}+\mathrm{C})$ content exceeds approximately $80 \%$ (Figure $4 \mathrm{a}$ ). We also tested to see whether the sizes of the MspI fragments generated influenced the counts obtained, as the 


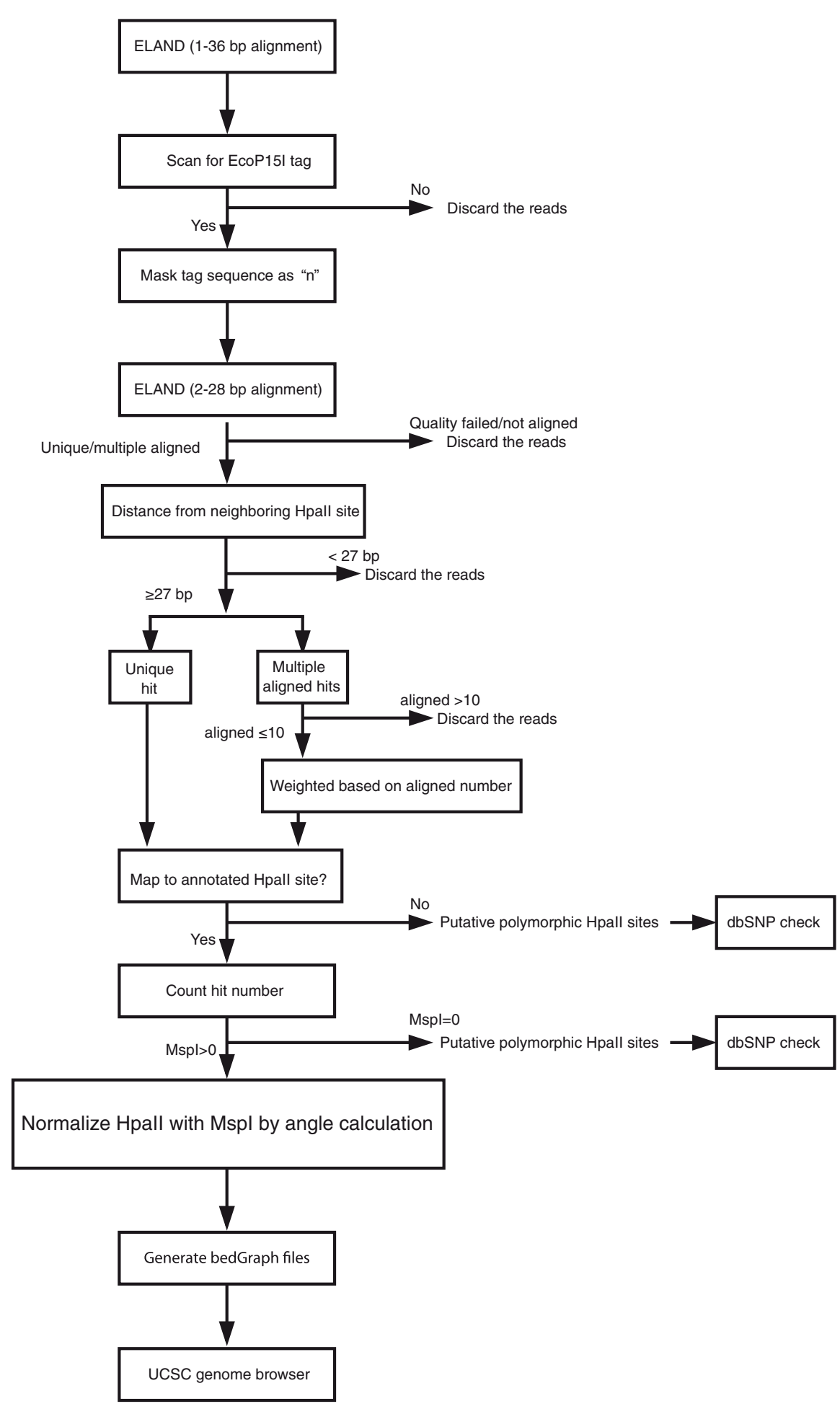

Figure 3 HELP-tagging analysis workflow. The analysis workflow for HELP-tagging data is illustrated. Only sequence reads that contain the adapter sequence and map to a single or $\leq 10$ sites are retained, the latter repetitive sequences distributed by weighting among the matched loci. Potential polymorphic loci are annotated. Normalization of Hpall by Mspl using the angle calculation described in the previous figure is performed and files are generated for genome browser visualization. UCSC, University of California Santa Cruz. 
(a)

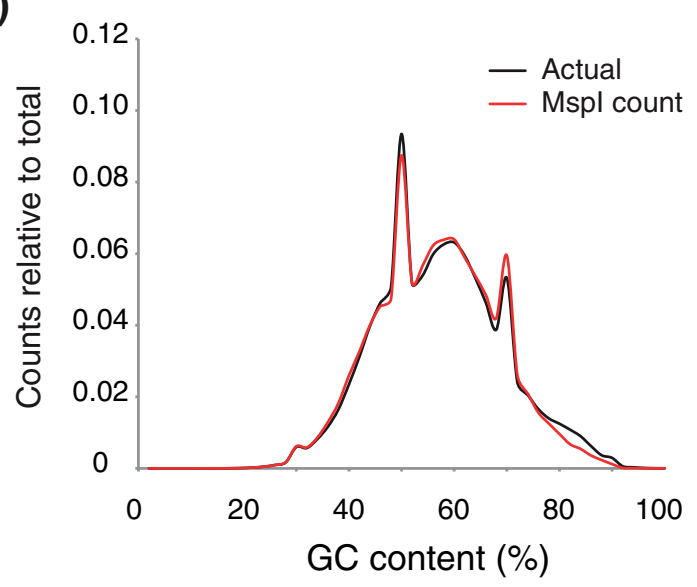

(b)

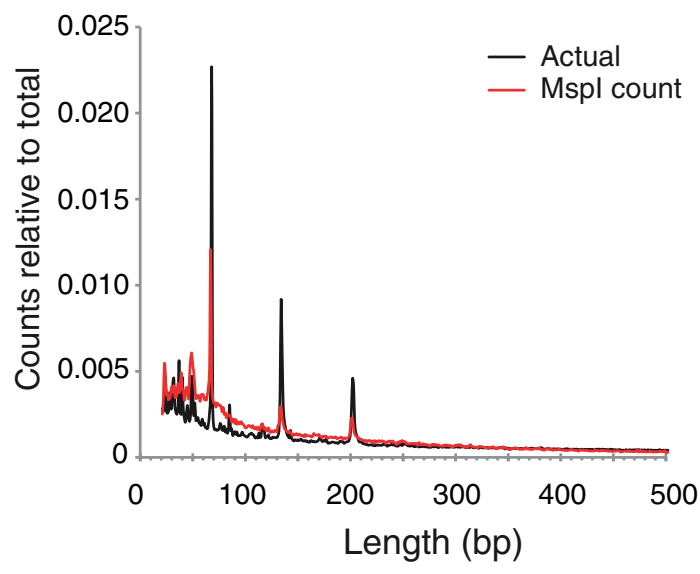

Figure 4 Base composition and fragment length influences on sequence counts. (a) The proportion of $(\mathrm{G}+\mathrm{C})$ nucleotides was calculated for the 50-bp sequence centered around each annotated CCGG in the reference human genome. The base composition of all of the Mspl sequences generated from the human ES cell line studied was also calculated. The relative proportion for $(\mathrm{G}+\mathrm{C})$ content in $2 \%$ bins for each set of data was calculated and plotted as shown. The black line shows the proportions in the reference genome, while the red line illustrates the distribution we observed in our Mspl experiment. Two peaks representing base composition in repetitive sequences are apparent. The Mspl distribution closely matches the expected distribution except when the base composition exceeds approximately $80 \%$, when it is slightly under-represented. (b) We calculated the relative frequencies of Mspl digestion product sizes in the human reference genome. In this case we found that the shorter fragments are more likely to be sequenced than larger ( $\geq 300 \mathrm{bp}$ ) fragments. The three major peaks observed represent Alu short interspersed repetitive element (SINE) sequences.

digestion by type III endonucleases like EcoP15I is most efficient when a pair of enzymes is present in convergent orientation on the same DNA molecule [19]. We find that there is indeed an over-representation for shorter $(\leq 300$ bp) and a corresponding modest under-representation for larger MspI fragments (Figure 4b).

\section{Identification of polymorphic CCGG sequences}

Whereas MSCC used MmeI and generates an 18- to 19bp sequence flanking the HpaII site [15], our use of EcoP15I generates a 27-bp flanking sequence. We asked whether this size difference influenced our ability to align sequences to the reference genome. We truncated our sequence reads to $19 \mathrm{bp}$ to mimic the MSCC read length and found that this caused a profound loss of ability to align reads unambiguously (Table S7 in Additional file 1). To compensate for the low alignment rate, the MSCC report described an ingenious strategy of alignment to the sequences immediately flanking the annotated HpaII sites in the reference genome [15], an approach sufficiently powerful that it generated the well-validated data that they described. However, it does not offer the possibility of identifying polymorphic HpaII sites at the high frequencies that we previously observed for our HELPseq assay [10]. We tested whether our longer sequences allowed the identification of loci at which an HpaII site is annotated in the reference genome but we obtain no sequence reads, and the opposite situation where we observed at least four MspI reads (the average number per annotated MspI/HpaII site) flanking a locus not annotated in the reference genome. In Table S8 in Additional file 1 we list approximately 6,600 candidate polymorphic HpaII sites, of which examples are shown in Figure 5, confirmed by targeted resequencing of those loci. The 6,600 loci were selected based on overlap with dbSNP entries, allowing us to evaluate the pattern of sequence variability at these loci. Approximately $80 \%$ of the SNPs are C:G to T:A transversions, consistent with deamination-mediated decay of methylcytosine being the cause of the polymorphism [23]. Polymorphic CG dinucleotides are major potential sources of error not only for microarrays, which are designed to a consensus genomic sequence, but also for both bisulfite sequencing, which would read the $\mathrm{C}$ to $\mathrm{T}$ transversion as unmethylated, and mass spectrometry-based assays, requiring the development of specific analytical approaches such as we have described [24].

\section{DNA methylation studies of human embryonic stem cells}

To test whether the HELP-tagging assay was generating data that are biologically plausible, we tested the methylation of different genomic sequence compartments as density plots of B angle values for the human ES cells used in these studies. In Figure S2a in Additional file 1 we show how promoters (defined as $-2 \mathrm{~kb}$ to $2 \mathrm{~kb}$ from the transcription start site of RefSeq genes), gene bodies (the remaining region within the RefSeq gene) and intergenic (all other) sequences compare, finding the expected enrichment of hypomethylated loci with larger B angle values in promoter regions. When we compared unique with repetitive sequences, again we found the expected 
(a)

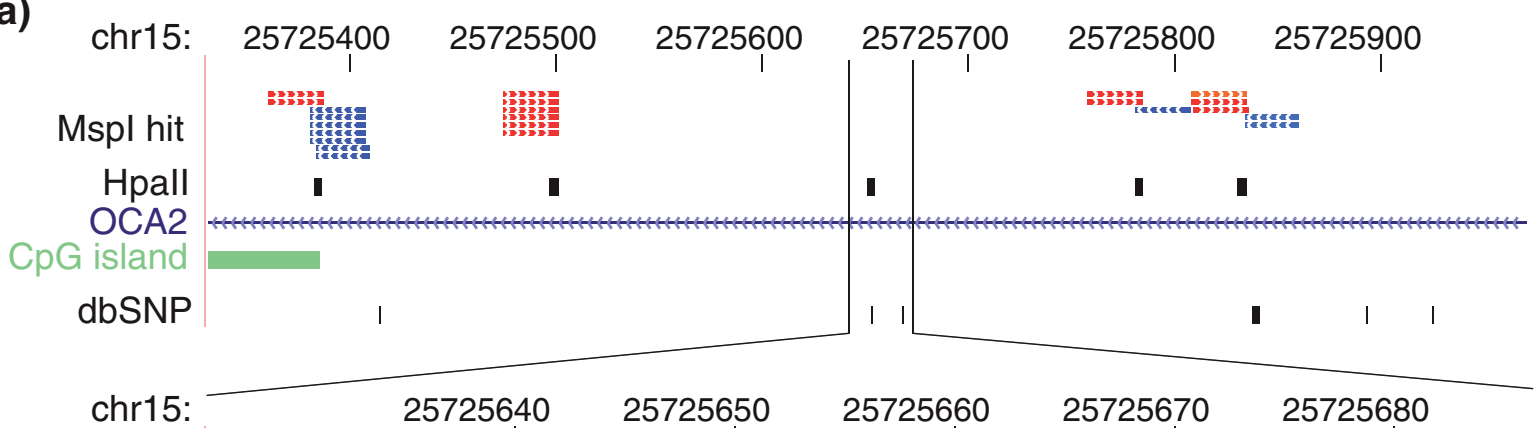

Reference AGT C T CT T CACTCTCACATTCTAGCCC GGGCTCCTGCCCACATTCTGCATGGCATGGCT

CA2

dbSNP

Hpall

Observed AGTCT CTTCACTCTCACATTCTAGCCC

rs12916836 rs12905726

A G CTCCTGCCCACACTCTGCATGG CATGGCC

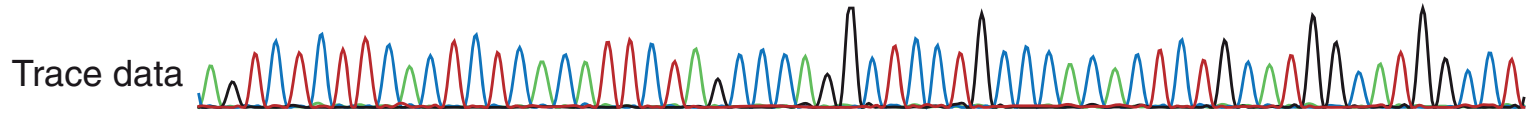

(b)
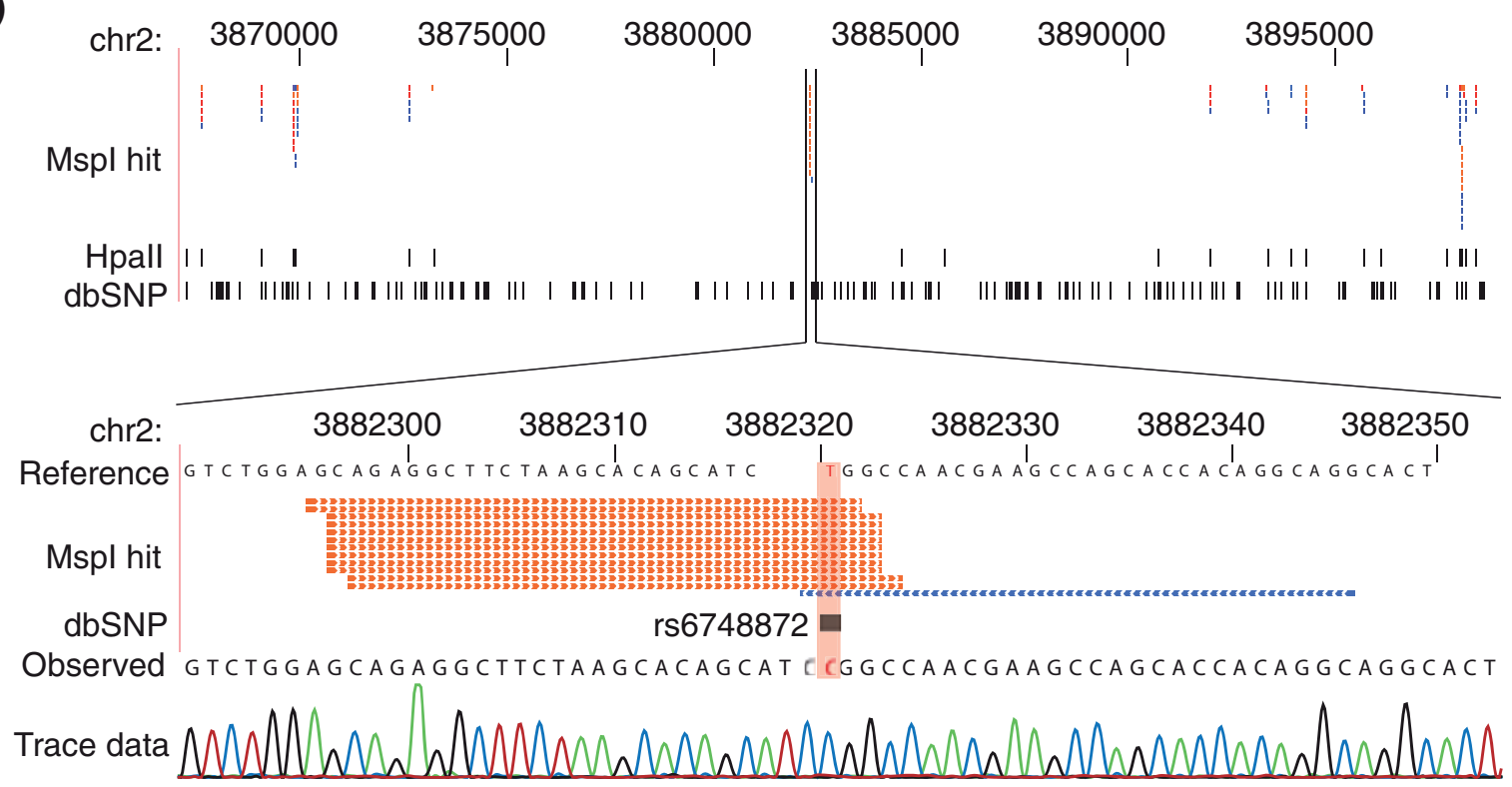

Figure 5 Polymorphic Hpall sites identified by HELP-tagging. Examples of Hpall sites (a) annotated in the reference genome sequence but not represented by Mspl reads or (b) not annotated in the reference human genome and represented by at least four Mspl reads are shown. In each case there is a SNP defined by dbSNP that indicates the C:T to G:A transversion that eliminates or restores the CCGG Hpall site.

pattern of increased methylation of repetitive DNA compared with unique sequences (Figure S2b in Additional file 1). Combining these observations, we tested whether the transposable element component of annotated repetitive DNA sequences showed any tendency to unusual methylation near gene promoters. In Figure 6 we show that while transposable elements are generally methylated and are depleted near gene promoters, those that are proximal to promoters tend to be less methylated than those located more distally. While many types of transposable elements were represented in this promoter-proximal hypomethylated group, we found a sub- 
(a)

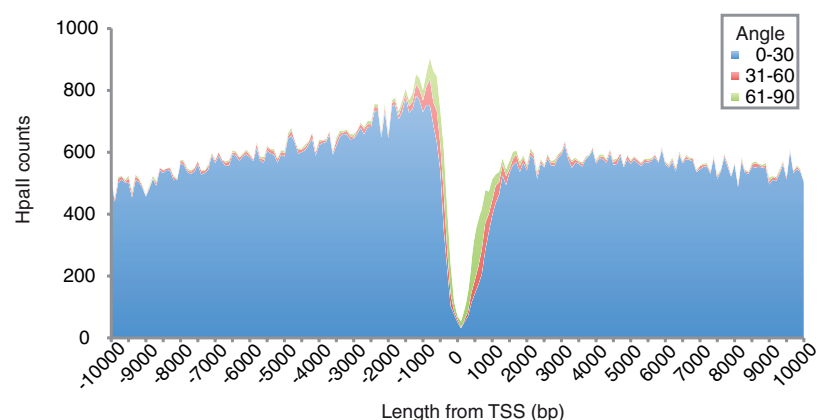

(b)

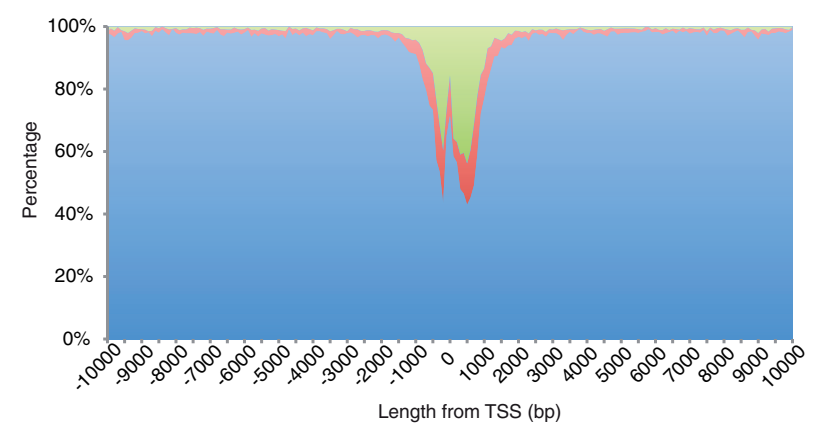

Figure 6 Identification of a position effect on DNA methylation in transposable elements located close to gene promoters. The distance from RefSeq gene transcription start sites and DNA methylation status are shown. The $x$-axis displays the distance from transcription start sites (TSSs). Hpall sites were categorized into three groups by angle, 0 to 30 (blue), 31 to 60 (red) and 61 to 90 (green)). (a) Number of Hpall sites; (b) proportions of each angle category (\%).

set to be the most markedly over-represented, as shown in Figure 6c.

The outcome of these studies was an improvement in the previously described MSCC [15] and HELP-seq [10] assays, not only by means of technical modifications such as the use of EcoP15I but also because of the concurrent use of MspI for normalization. The effect of these modifications was not only to increase the accuracy of the assay but also to enhance the ability to align sequences to the genome and thus identify polymorphic HpaII/MspI sites. The means of normalization of HpaII by MspI using an angular metric is an innovation that improved the data accuracy substantially and may have applications in other MPS assay normalization strategies. We were also able to discard reads that did not contain the expected adapter sequences, and created a straightforward data analytical pipeline that will facilitate processing of these HELP-tagging data by others.

The potential sources of systematic artifacts due to base composition or digestion product size were evaluated. Apart from a modest decrease in representation in regions above approximately $80 \%(\mathrm{G}+\mathrm{C})$ content, base composition did not cause biases in representations, possibly in part due to our avoidance of a gel purification step in library preparation [22]. Fragment length does influence the outcome, most likely due to effects on EcoP15I digestion [19], although the effects should be similar for both HpaII and MspI and should, therefore, largely cancel each other out in the normalization step. It is possible that endogeneous EcoP15I sites could influence the representations, but to have an effect they would have to be located within the $27 \mathrm{bp}$ adjacent to HpaII/MspI sites and would cause digestion of the ligated adapter, causing those loci to be under-represented in both HpaII and MspI datasets. The most likely effect of these endoge- neous sites is that they contribute to the proportion of loci at which we could not obtain sequence reads.

Our exploration of the distribution of cytosine methylation in the same human ES cell line studied by Lister $e t$ al. [6] showed consistent results, with hypomethylation of transcription start sites and methylation of transposable elements, as expected from long-standing observations in the field. We furthermore discovered a limited subset of transposable elements that is hypomethylated when in close proximity to transcription start sites. When this subset was studied to determine whether certain types of transposable elements were disproportionately over-represented, we found two broad classes, one of transposable element fossils with no innate capacity to replicate themselves (the ancient DNA, long interspersed repetitive elements (LINEs) and short interspersed repetitive elements (SINEs) shown in Figure 6c) and younger ERV1 long terminal repeat retroelements. Loss of methylation of functionally inactive transposable elements is likely to be of no negative consequence to the host genome, consistent with the host defense hypothesis [25], while the young ERV long terminal repeats represent a group of transposons whose function has been harnessed as promoters of endogeneous genes [26,27]. This observation demonstrates the value of a high-resolution, genome-wide assay like HELP-tagging to define potential functional elements in an unbiased manner.

\section{Conclusions}

We propose that MPS-based assays such as RRBS [12], MSCC [15] and HELP-tagging will prove to be the assays of choice for epigenome-wide association studies in human disease, with the latter two preferable as we begin to explore the CG-depleted majority of the genome. It should not be necessary to run MspI assays every time a HELP-tagging assay is performed, suggesting that a com- 
mon MspI dataset can serve as a universal reference for a species, allowing a single lane of Illumina sequencing of the HpaII library to provide the methylation data for that sample. The development of analytical pipelines to support analysis of these datasets will be critical to the success of these projects, while the careful ongoing assessment of potential sources of bias will also be essential for improving assay performance.

\section{Materials and methods}

\section{Cell preparation and DNA purification}

H1 human ES cells (NIH code WA01 from Wicell Research Institute, Madison, WI, USA) were cultured on matrigel (BD Biosciences, San Diego, CA, USA), at $37^{\circ} \mathrm{C}$, $5 \% \mathrm{O}_{2}$ and $5 \% \mathrm{CO}_{2}$. Amplified human ES cell pluripotency was assessed by flow cytometry with SSEA4, CD24 and Oct4 markers. To extract DNA, the cells were suspended in $10 \mathrm{ml}$ of a solution of $10 \mathrm{mM}$ Tris- $\mathrm{HCl}(\mathrm{pH}$ 8.0), $0.1 \mathrm{M}$ EDTA and $1 \mathrm{ml}$ of $10 \%$ SDS to which $10 \mu \mathrm{l}$ of RNase A $(20 \mathrm{mg} / \mathrm{ml})$ was added. After incubation for 1 hour at $37^{\circ} \mathrm{C}, 50 \mu \mathrm{l}$ of proteinase $\mathrm{K}(20 \mathrm{mg} / \mathrm{ml})$ was added and the solution was gently mixed and incubated in a $50^{\circ} \mathrm{C}$ water bath overnight. To purify the lysate, it was extracted three times using saturated phenol, then twice with chloroform, and dialyzed for 16 hours at $4^{\circ} \mathrm{C}$ against three changes of $0.2 \times$ SSC. Following dialysis, the DNA was concentrated by coating the dialysis bags in polyethylene glycol (molecular weight 20,000). The purity and final concentration of the purified DNA was checked by spectrometry (Nanodrop, Wilmington, DE, USA).

\section{Illumina library preparation}

The sample preparation steps are illustrated in Figure 1. Two custom adapters were created for HELP-tagging, referred to as $\mathrm{AE}$ and $\mathrm{AS}$. As well as an Illumina adapter sequence, adapter $\mathrm{AE}$ contains an EcoP15I recognition site and a T7 promoter sequence. Adapter AS contains an Illumina sequencing primer sequence. The adapter and primer sequences for library preparation are listed in Table S9 in Additional file 1. Genomic DNA $(5 \mu \mathrm{g})$ was digested with HpaII and MspI in separate $200 \mu \mathrm{l}$ reactions and purified by phenol/chloroform extraction followed by ethanol precipitation. The digested genomic DNA was ligated to adapter AE using a New England Biolabs Quick Ligation Kit $(25 \mu \mathrm{l}$ of $2 \times$ Quick ligase buffer, $3 \mu \mathrm{g}$ of HpaII-digested DNA or $1 \mu \mathrm{g}$ of MspI-digested DNA, 0.1 $\mu \mathrm{l}$ of Adapter $\mathrm{AE}(1 \mu \mathrm{M}), 3 \mu \mathrm{l}$ of Quick Ligase in a final volume of $50 \mu \mathrm{l})$. After AE ligation, the products were purified using Agencourt AMpure beads (Beckman Coulter, Brea CA, USA), then digested with EcoP15I (New England Biolabs). The restriction fragments were end-repaired to inhibit to dimerization of adapters, and tailed with a single $\mathrm{dA}$, at the $3^{\prime}$ end. After the $\mathrm{dA}$ tailing reaction, adapter AS was ligated to the dA-tailed frag- ments using a New England Biolabs Quick Ligation Kit (25 $\mu$ l of $2 \times$ Quick ligase buffer, $2.5 \mu \mathrm{l}$ of adapter AS (10 $\mu \mathrm{M}), 2.5 \mu \mathrm{l}$ of Quick Ligase in a final volume $50 \mu \mathrm{l})$. After ligation, products were purified using the MinElute PCR purification kit (Qiagen, Hilden, Germany) and in vitrotranscribed using the Ambion MEGAshortscriptkit (Life Technologies, Carlsbad, CA, USA). Following in vitro transcription, products were purified with an RNeasy clean-up kit (Qiagen) before reverse transcription was performed using the Invitrogen SuperScript III kit (Life Technologies). The first strand cDNA produced was used as a template for PCR using the following conditions: $96^{\circ} \mathrm{C}$ for 2 minutes, then 18 cycles of $96^{\circ} \mathrm{C}$ for 15 seconds and $72^{\circ} \mathrm{C}$ for 15 seconds followed by 5 minutes at $72^{\circ} \mathrm{C}$ for the final extension. After PCR, the library was purified using a QIAQuick PCR clean-up kit (Qiagen).

\section{Single-locus quantitative validation assays}

Bisulfite conversion and MassArray (Sequenom, San Diego, CA, USA) were performed using an aliquot of the same sample of DNA as was used for the high-throughput assays described above. Bisulfite conversion was performed with an EZ DNA Methylation kit (Zymo Research, Orange, CA, USA). Bisulfite primers were designed using MethPrimer [28], specifying the desired product length (250 to $450 \mathrm{bp}$ ), primer length (23 to 29 bp) and primer $\mathrm{Tm}$ (56 to $62^{\circ} \mathrm{C}$ ). PCR was performed using FastStart High Fidelity Taq polymerase (Roche, Basel, Switzerland) with the following conditions: $95^{\circ} \mathrm{C}$ for 10 minutes, then 42 cycles of $95^{\circ} \mathrm{C}$ for 30 seconds, primer-specific $\mathrm{Tm}$ for 30 seconds and $72^{\circ} \mathrm{C}$ for 1 minute, followed by $72^{\circ} \mathrm{C}$ for 10 minutes for the final extension. Primer-specific $\mathrm{Tm}$ and sequence information are provided in Table S6 in Additional file 1. Bisulfite MassArray assays were performed by the institutional Genomics Core Facility. The data were analyzed using the analytical pipeline we have previously described [24].

\section{Bioinformatic analysis}

Four lanes of sequencing were performed using an Illumina GA IIx Sequencer at the institutional Epigenomics Shared Facility. Three lanes were used for technical replicates of MspI, for the methylation-insensitive reference dataset. Images generated by the Illumina sequencer were analyzed by Illumina pipeline software (versions 1.3 to 1.4). Initial data processing was performed using the default read length of $36 \mathrm{bp}$, after which we isolated the sequences in which we found adapter sequences on the 3 '-end, replaced the adapter sequence with a poly $(\mathrm{N})$ sequence of the same length, and re-ran the Illumina ELAND pipeline again on these sequences with the sequence length set at $27 \mathrm{bp}$ (the 2 to $28 \mathrm{bp}$ subsequence). The data within the ELAND_extended.txt files were used for counting the number of aligned sequences adjacent to 
each CCGG (HpaII/MspI) site annotated in the hg18 freeze of the human genome at the UCSC genome browser. We permitted up to two mismatches in each sequence, and allowed a sequence to align to up to a maximum of 10 locations within the genome. For non-unique alignments, a sequence was assigned a partial count for each alignment location amounting to $1 / \mathrm{n}$, where $\mathrm{n}$ represents the total number of aligned positions. To normalize the data between experiments, the number of sequences associated with each HpaII site was divided by the total number of sequences (including partial counts) aligning to all HpaII sites in the same sample. We refer to this figure as the fixed count below.

To examine an influence of $(\mathrm{G}+\mathrm{C})$ mononucleotide content on counts of sequences obtained, we extracted the $(\mathrm{G}+\mathrm{C})$ annotation from the hg18 freeze of the human genome at UCSC and examined the distribution of sequence counts according to $(\mathrm{G}+\mathrm{C})$ content. Annotated percentages of $(\mathrm{G}+\mathrm{C})$ content were available for adjacent 5-bp windows. For each annotated HpaII site, we calculated the mean percentage $(\mathrm{G}+\mathrm{C})$ for a 50 -bp region centered at the restriction site. Counts of sequences associated with HpaII sites were obtained for 50 sequential non-overlapping windows of $2 \%(\mathrm{G}+\mathrm{C})$ (the minimum possible in a sample of 50-bp regions). These data were then normalized as a proportion of the total number of fragments. Comparisons were made to the expected frequencies, which for each $2 \%(\mathrm{G}+\mathrm{C})$ bin was represented by the counts of HpaII sites falling within a range relative to the total number of HpaII sites in the genome. This analysis was performed on both HpaII and MspIdigested DNA for comparison.

The potential effect of distance between HpaII sites on sequences counts obtained at each HpaII site was measured by summing the counts of sequences aligning within each restriction fragment, and normalizing the result with respect to total sequence count. As with the $(\mathrm{G}+\mathrm{C})$ analysis above, this was performed for both MspI and Hpall digested restriction fragments. The data were compared with the expected distribution determined by performing virtual restriction digestion using genomic HpaII site coordinates, and normalizing the number of virtual fragments of each size with respect to the total number of these virtual fragments.

\section{Additional material}

Additional file 1 Supplemental data containing two figures (Figures $\mathrm{S}$ and S2) and nine tables (Tables S1 to S9).

\section{Abbreviations}

bp: base pair; CG/CpG: cytosine-guanine dinucleotide; ChIP: chromatin immunoprecipitation; ES: embryonic stem; $(\mathrm{G}+\mathrm{C})$ : guanine and cytosine mononucleotides; HELP: Hpall tiny fragment Enrichment by Ligation-mediated PCR; MPS: massively-parallel sequencing; MSCC: methyl-sensitive cut counting; RRBS: reduced representation bisulfite sequencing; UCSC: University of California Santa Cruz

\section{Authors' contributions}

MS and JMG designed the assays and strategies for its analysis, MS performed all library preparation and characterization, MS, DL and MP performed bisulfite validation studies, while QJ and AMCL performed computational analyses. JMG and MS prepared the manuscript.

\section{Acknowledgements}

This work is supported by a grant from the National Institute of Health $(\mathrm{NIH}$ R01 HG004401) to JMG. The authors thank Shahina Maqbool PhD, Raul Olea and Gael Westby of the Einstein Epigenomics Shared Facility for their contributions, Drs Eric Bouhassira and Emmanuel Olivier (Einstein) for the WA01/H1 human ES cell line, and Einstein's Center for Epigenomics.

\section{Author Details}

Department of Genetics (Computational Genetics), Center for Epigenomics, Albert Einstein College of Medicine, 1301 Morris Park Avenue, Bronx, NY 10461, USA

Received: 8 January 2010 Revised: 16 March 2010

Accepted: 1 April 2010 Published: 1 April 2010

\section{References}

1. Esteller M: Epigenetics in cancer. N Engl J Med 2008, 358:1 148-1159.

2. Kopelovich L, Crowell JA, Fay JR: The epigenome as a target for cancer chemoprevention. J Nat/ Cancer Inst 2003, 95:1747-1757.

3. Zilberman D, Gehring M, Tran RK, Ballinger T, Henikoff S: Genome-wide analysis of Arabidopsis thaliana DNA methylation uncovers an interdependence between methylation and transcription. Nat Genet 2007, 39:61-69.

4. Cokus SJ, Feng S, Zhang X, Chen Z, Merriman B, Haudenschild CD, Pradhan S, Nelson SF, Pellegrini M, Jacobsen SE: Shotgun bisulphite sequencing of the Arabidopsis genome reveals DNA methylation patterning. Nature 2008, 452:215-219.

5. Lister R, O'Malley RC, Tonti-Filippini J, Gregory BD, Berry CC, Millar AH, Ecker JR: Highly integrated single-base resolution maps of the epigenome in Arabidopsis. Cell 2008, 133:523-536.

6. Lister R, Pelizzola M, Dowen RH, Hawkins RD, Hon G, Tonti-Filippini J, Nery JR, Lee L, Ye Z, Ngo QM, Edsall L, Antosiewicz-Bourget J, Stewart R, Ruotti V, Millar AH, Thomson JA, Ren B, Ecker JR: Human DNA methylomes at base resolution show widespread epigenomic differences. Nature 2009, 462:315-322.

7. Heijmans BT, Tobi EW, Stein AD, Putter H, Blauw GJ, Susser ES, Slagboom PE, Lumey LH: Persistent epigenetic differences associated with prenatal exposure to famine in humans. Proc Natl Acad Sci USA 2008, 105:17046-17049.

8. Yamashita S, Hosoya K, Gyobu K, Takeshima H, Ushijima T: Development of a novel output value for quantitative assessment in methylated DNA immunoprecipitation-CpG island microarray analysis. DNA Res 2009, 16:275-286

9. Down TA, Rakyan VK, Turner DJ, Flicek P, Li H, Kulesha E, Graf S, Johnson N, Herrero J, Tomazou EM, Thorne NP, Backdahl L, Herberth M, Howe KL, Jackson DK, Miretti MM, Marioni JC, Birney E, Hubbard TJ, Durbin R, Tavare S, Beck S: A Bayesian deconvolution strategy for immunoprecipitationbased DNA methylome analysis. Nat Biotechnol 2008, 26:779-785.

10. Oda M, Glass JL, Thompson RF, Mo Y, Olivier EN, Figueroa ME, Selzer RR, Richmond TA, Zhang X, Dannenberg L, Green RD, Melnick A, Hatchwell E, Bouhassira EE, Verma A, Suzuki M, Greally JM: High-resolution genomewide cytosine methylation profiling with simultaneous copy number analysis and optimization for limited cell numbers. Nucleic Acids Res 2009, 37:3829-3839.

11. Ordway JM, Bedell JA, Citek RW, Nunberg AN, Jeddeloh JA: MethylMapper: a method for high-throughput, multilocus bisulfite sequence analysis and reporting. Biotechniques 2005, 39:. 464, 466, 468 passim.

12. Meissner A, Mikkelsen TS, Gu H, Wernig M, Hanna J, Sivachenko A, Zhang X, Bernstein BE, Nusbaum C, Jaffe DB, Gnirke A, Jaenisch R, Lander ES: Genome-scale DNA methylation maps of pluripotent and differentiated cells. Nature 2008, 454:766-770. 
13. t Hoen PA, Ariyurek $Y$, Thygesen $H H$, Vreugdenhil E, Vossen $R H$, de Menezes RX, Boer JM, van Ommen GJ, den Dunnen JT: Deep sequencingbased expression analysis shows major advances in robustness, resolution and inter-lab portability over five microarray platforms. Nucleic Acids Res 2008, 36:e141.

14. Brunner AL, Johnson DS, Kim SW, Valouev A, Reddy TE, Neff NF, Anton E, Medina C, Nguyen L, Chiao E, Oyolu CB, Schroth GP, Absher DM, Baker JC, Myers RM: Distinct DNA methylation patterns characterize differentiated human embryonic stem cells and developing human fetal liver. Genome Res 2009, 19:1044-1056.

15. Ball MP, Li JB, Gao Y, Lee JH, LeProust EM, Park IH, Xie B, Daley GQ, Church GM: Targeted and genome-scale strategies reveal gene-body methylation signatures in human cells. Nat Biotechnol 2009, 27:361-368.

16. Fazzari MJ, Greally JM: Epigenomics: beyond CpG islands. Nat Rev Genet 2004, 5:446-455.

17. Hattori N, Nishino K, Ko YG, Hattori N, Ohgane J, Tanaka S, Shiota K: Epigenetic control of mouse Oct-4 gene expression in embryonic stem cells and trophoblast stem cells. J Biol Chem 2004, 279:17063-17069.

18. Irizarry RA, Ladd-Acosta C, Wen B, Wu Z, Montano C, Onyango P, Cui H, Gabo K, Rongione M, Webster M, Ji H, Potash JB, Sabunciyan S, Feinberg AP: The human colon cancer methylome shows similar hypo- and hypermethylation at conserved tissue-specific $\mathrm{CpG}$ island shores. Nat Genet 2009, 41:178-186.

19. Moncke-Buchner E, Rothenberg M, Reich S, Wagenfuhr K, Matsumura $\mathrm{H}$, Terauchi R, Kruger DH, Reuter M: Functional characterization and modulation of the DNA cleavage efficiency of type III restriction endonuclease EcoP15I in its interaction with two sites in the DNA target. J Mol Biol 2009, 387:1309-1319.

20. Greallylab [http://greallylab.aecom.yu.edu/HELP-tagging/

21. Dohm JC, Lottaz C, Borodina T, Himmelbauer H: Substantial biases in ultra-short read data sets from high-throughput DNA sequencing. Nucleic Acids Res 2008, 36:e105.

22. Quail MA, Kozarewa I, Smith F, Scally A, Stephens PJ, Durbin R, Swerdlow $H$, Turner DJ: A large genome center's improvements to the lllumina sequencing system. Nat Methods 2008, 5:1005-1010.

23. Duncan BK, Miller JH: Mutagenic deamination of cytosine residues in DNA. Nature 1980, 287:560-561

24. Thompson RF, Suzuki M, Lau KW, Greally JM: A pipeline for the quantitative analysis of $C G$ dinucleotide methylation using mass spectrometry. Bioinformatics 2009, 25:2164-2170.

25. Yoder JA, Walsh CP, Bestor TH: Cytosine methylation and the ecology of intragenomic parasites. Trends Genet 1997, 13:335-340.

26. Conley AB, Piriyapongsa J, Jordan IK: Retroviral promoters in the human genome. Bioinformatics 2008, 24:1563-1567.

27. Cohen CJ, Lock WM, Mager DL: Endogenous retroviral LTRs as promoters for human genes: a critical assessment. Gene 2009, 448:105-114.

28. MethPrimer [http://www.urogene.org/methprimer/]

doi: 10.1186/gb-2010-11-4-r36

Cite this article as: Suzuki et al., Optimized design and data analysis of tagbased cytosine methylation assays Genome Biology 2010, 11:R36

Submit your next manuscript to BioMed Central and take full advantage of:

- Convenient online submission

- Thorough peer review

- No space constraints or color figure charges

- Immediate publication on acceptance

- Inclusion in PubMed, CAS, Scopus and Google Scholar

- Research which is freely available for redistribution

Submit your manuscript at www.biomedcentral.com/submit
C Biomed Central 